\title{
Efecto de la Profundidad sobre la Soldabilidad de Aceros Ferríticos en Ambientes Simulados Unidos por Soldadura Húmeda
}

Fernando Macías López ${ }^{1}$, Luciano Eliezer Ramírez Vidaurri² ${ }^{2}$ Jorge Leobardo Acevedo Dávilaํㅜ, Héctor M. Hdz-García ${ }^{1}$, A. Hernández-Rodríguez ${ }^{1}$, J. Jorge Ruiz Mondragon ${ }^{1}$

${ }^{1}$ Corporación Mexicana de Investigación en Materiales S.A de C.V., Technological Development Department, Saltillo, Coahuila, México.

2 Instituto Tecnológico de Saltillo - Simulation and Solidification, Saltillo, Coahuila, México.

Recibido: 13 Nov., 2015

Aprobado: 07 Enero, 2016

E-mails: fmacias@comimsa.com (FML), elramirez@its.mx (LERV), jacevedo@ comimsa.com (JLAD), hmanuelhdz@ comimsa.com (HMH-G), alejandra. hdz@comimsa.com (AH-R), jjorge. ruiz@comimsa.com (JJRM)
Resumen: El objetivo de este trabajo fue evaluar el efecto de la profundidad sobre las propiedades en soldaduras de ranura en " $\mathrm{V}$ " con varios cordones de relleno. Metal base y electrodos comerciales fueron utilizados (ASTM A36 y AWS E7014) y cuatro diferentes condiciones se utilizaron para desarrollar las soldaduras húmedas como son 10, 20, 30 y 40m de profundidad. Una cámara presurizada con 30 atmósferas de capacidad fue utilizada para simular la profundidad, inundada con agua fresca. Un buzo soldador fue quien realizo las soldaduras. Las propiedades en la sección transversal mediante pruebas de tenacidad CVN (Charpy V-notch), macro ataque y esfuerzo a la tensión fueron determinadas por correlación. Las pruebas mecánicas con mejores resultados se observaron en la condición de $20 \mathrm{~m}$ de profundidad.

Palabras Clave: Soldadura húmeda; Simulación; Microestructura.

\section{Weldability Depth Effect of Ferritic Steels in Wet Welding Simulated Environment}

\begin{abstract}
The objective of this work is to evaluate the influence of depth variation on multipass V-groove weld metal properties. Commercial base metal and covered electrodes (ASTM A36 and E7014) and four different conditions were used to perform the wet welds at 10, 20, 30 and 40 m depth. A pressurized chamber with 30 atmospheres capacity filled with water was used to simulate the depth. A certified dive welder was used to perform the welds. The properties in transverse cross-sections, Charpy V-notch tests, macro etching tests and tensile tests were determined for correlation. Mechanical tests showed better properties in the samples extracted from the $20 \mathrm{~m}$ depth condition.
\end{abstract}

Key-words: Wet welding; Simulation; Microstructure.

\section{Introducción}

La microestructura final obtenida en cualquier depósito de soldadura depende principalmente de la composición química, del consumible, de la velocidad de enfriamiento, de la energía lineal y de la templabilidad [1]. Debido a lo anterior los procesos de soldadura resultan ser uno de los principales causantes de defectos, ocasionados por la introducción de un alto contenido de calor de entrada el cual genera gradientes de temperatura, deformaciones plásticas y transformaciones de fase en el material soldado y por consecuencia, la formación de patrones de esfuerzos sobre la zona de fusión (ZF) y la zona afectada por el calor (ZAC) [2]. La soldadura húmeda ha sido reconocida como una de las técnicas más comunes para la construcción y reparación de estructuras costa afuera, como son plataformas marinas y tuberías de conducción, etc. Siendo este el más eficiente y de bajo costo, obteniendo gran popularidad en los trabajos de exploración y producción de hidrocarburos a mar abierto en el sector energético [3]. Una de las principales limitantes de la soldadura húmeda se encuentra en la disminución de las propiedades de resistencia y tenacidad en comparación con la soldadura realizada en superficie debido al contenido de Mn que disminuye mientras que el Si incrementa conforme aumenta la profundidad. Como lo comprobaron en sus investigaciones Christensen et al. [4], en donde mencionan que las variaciones en los contenidos de $\mathrm{Mn}$ y $\mathrm{C}$ afectan la resistencia en la soldadura, mientras que el contenido de $\mathrm{O}$ modifica la tenacidad en el metal de soldadura. 
Efecto de la Profundidad sobre la Soldabilidad de Aceros Ferríticos en Ambientes Simulados Unidos por

Soldadura Húmeda

En las soldaduras húmedas, las cantidades de $\mathrm{O}$ presentes en el metal líquido de soldadura son mayores debido a la disociación del agua en el arco eléctrico [5].

El aumento del contenido de Si mejora el porcentaje de fractura dúctil/frágil, al incrementar desde un 50\% hasta un $100 \%$ dúctil. Sin embargo, la pérdida de $\mathrm{Mn}$ por debajo de $0.30 \%$ e. p., afecta directamente el valor del esfuerzo último a la tensión (UTS) hasta en un 15\%. Ibarra et al. [6] explican que las soldaduras húmedas presentan propiedades mecánicas inferiores a las realizadas en superficie por la reacción química del O, H y C.

Con el aumento de la profundidad (presión) disminuye la templabilidad debido a la disminución del contenido de elementos aleantes y del aumento en el contenido de $\mathrm{O}$ [7]. El incremento de la profundidad aumenta la velocidad de enfriamiento [8] afectando directamente el tipo y el tamaño de las fases secundarias formadas, cuanto mayor es la velocidad de enfriamiento, más corto es el tiempo de solidificación y más fina es la estructura celular o dendrítica. Según la fase en que precipite dará como resultado las propiedades mecánicas de la unión soldada [9], esto debido a que la austenita puede transformarse en otras fases en base al tiempo y a la temperatura. Mecanismos que alteran la microestructura causando una disminución en las propiedades mecánicas de la unión soldada [10]. El hecho de la presión ejercida, la descomposición del agua, el rápido enfriamiento, el calor de entrada, el tipo de recubrimiento del electrodo y las variables del proceso de soldadura húmeda son factores responsables de muchos problemas que afectan la calidad de la unión.

Debido a lo anterior, el objetivo de este trabajo es describir la variación observada en las propiedades, químicas, mecánicas y en la distribución micro estructural.

\section{Procedimiento Experimental}

\subsection{Materia prima y preparación de las placas de acero pre-soldadura}

Se utilizaron cuatro placas de acero de bajo carbono de espesores de $12.7 \mathrm{~mm}$ con un esfuerzo de cedencia (SMYS) de 220 MPa de acuerdo a la especificación ASTM A36 [11]. La composición química de las placas de acero se resume en la Tabla 1. Así mismo, se emplearon electrodos comerciales de acuerdo a la especificación AWS A5.1 [12], clasificación 7014 de 3 mm de diámetro x 350 mm de longitud, marca Broco Souft Toch.

Tabla 1. Composición química de la placa de acero de bajo carbono utilizada.

\begin{tabular}{ccccccc}
\hline & \multicolumn{5}{c}{ Designación (\% e. p) } \\
ASTM A36 & $\mathbf{C}$ & Mn & $\mathbf{P}$ (máx.) & $\mathbf{S}$ (máx.) & Si (máx.) & Cu (mín.) \\
& 0,25 & --- & 0,04 & 0,05 & 0,4 & 0,20 \\
\hline
\end{tabular}

Las Tablas 2, 3 y 4 muestran las propiedades mecánicas y la composición química del material base y de los electrodos, respectivamente. Posteriormente, se prepararon cupones de $200 \mathrm{~mm}$ de ancho $\times 200 \mathrm{~mm}$ de longitud para ser unidos a tope con ranuras en " $\mathrm{V}$ " a $70^{\circ}$ (Figura 1). Adicionalmente, se colocaron soleras de respaldo de $5 \mathrm{~mm}$ de espesor $\times 25 \mathrm{~mm}$ de ancho, las dimensiones de la abertura de raíz utilizada fueron de $2.66 \mathrm{~mm}$.

Tabla 2. Especificaciones y propiedades mecánicas del metal base (acero bajo carbono).

\begin{tabular}{ccc} 
Grado de acero & $\begin{array}{c}\text { Resistencia a la tensión, Ultimate Tensile } \\
\text { Strength* (MPa) }\end{array}$ & $\begin{array}{c}\text { Esfuerzo de cedencia Specified Minimum } \\
\text { Yield Strength** }\end{array}$ \\
ASTM A36 & $400-550$ & 250 \\
\hline
\end{tabular}

*UTS: Ultimate Tensile Strength. ${ }^{* *}$ SMYS: Specified Minimum Yield Strength.

Tabla 3. Composición química del material de aporte (AWS A5.1).

\begin{tabular}{ccccccccc}
\hline & \multicolumn{8}{c}{ Designación (\% e. p.) } \\
& $\mathbf{C}$ & $\mathbf{S i}$ & $\mathbf{M n}$ & $\mathbf{P}$ & $\mathbf{S}$ & $\mathbf{C u}$ & $\mathbf{A l}$ & $\mathbf{C r}$ \\
AWS & 0,072 & 0,244 & 1,185 & 0,012 & 0,002 & 0,016 & 0,024 & 0,024 \\
\cline { 2 - 10 } E7014 & $\mathbf{M o}$ & $\mathbf{N i}$ & $\mathbf{V}$ & $\mathbf{T i}$ & $\mathbf{N b}$ & $\mathbf{C o}$ & $\mathbf{F e}$ & \\
& 0,005 & 0,023 & 0,015 & 0,008 & 0,017 & 0,009 & Bal. \\
\hline
\end{tabular}


Tabla 4. Especificaciones y propiedades mecánicas del material de aporte (AWS A5.1).

\begin{tabular}{cccc} 
Fabricante & Especificación AWS & Clasificación AWS & $\begin{array}{c}\text { Resistencia última a la tensión } \\
\text { (MPa) }\end{array}$ \\
Broco & $\mathrm{A} 5,1$ & $\mathrm{E} 7014$ & 482 \\
\hline
\end{tabular}

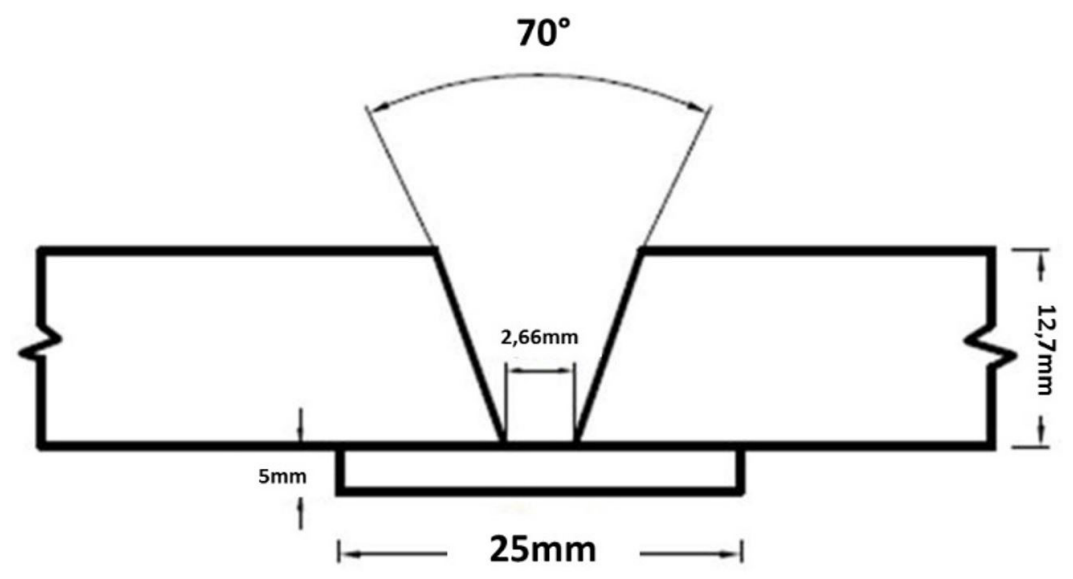

Figura 1. Junta con preparación de bisel en "V" con respaldo para la aplicación de soldaduras húmedas en material ASTM A36 a 40m de profundidad.

\subsection{Soldadura aplicada a los cupones de acero ASTM A36 a diferentes profundidades}

Las soldaduras fueron aplicadas en ambientes simulados a diferentes profundidades: 10m, 20m, 30m y 40m. En este caso, el buzo soldador requirió de una cámara presurizada inundada de agua no salina. Los parámetros utilizados en esta investigación se muestran en la Tabla 5.

Cabe mencionar que se utilizó una corriente directa electrodo al positivo (DCEP) empleando una velocidad de avance de $\sim 300$ - $500 \mathrm{~mm} / \mathrm{min}$ en función de la profundidad. Un promedio de 15 cordones de soldadura y 5 camas fueron requeridas para cubrir el espesor de la unión de $12.7 \mathrm{~mm}$. Modificándose las variables de operación mostradas en la Tabla 5.

Tabla 5. Parámetros utilizados para las uniones realizadas por el proceso de soldadura húmeda en aceros ASTM A36 con aporte AWS E7014.

\begin{tabular}{cccccc}
\hline Muestra & $\begin{array}{c}\text { Clasificación } \\
\text { AWS }\end{array}$ & $\begin{array}{c}\text { Profundidad } \\
(\mathbf{m})\end{array}$ & $\begin{array}{c}\text { Voltaje } \\
\text { (V) }\end{array}$ & $\begin{array}{c}\text { Corriente } \\
\text { (A) }\end{array}$ & $\begin{array}{c}\text { Velocidad de avance } \\
\text { (mm/min) }\end{array}$ \\
\hline 1 & E7014 & 10 & 30 & 158,2 & 418,7 \\
2 & E7014 & 20 & 32 & 162,0 & 337,4 \\
3 & E7014 & 30 & 34 & 171,8 & 549,0 \\
4 & E7014 & 40 & 36 & 162,0 & 370,6 \\
\hline
\end{tabular}

\subsection{Evaluación mecánica y química de los cordones de soldadura}

Las pruebas mecánicas se realizaron de acuerdo a la norma AWS D3.6. [13], (Figura 2). Las probetas de tensión se maquinaron con dimensiones de $200 \mathrm{~mm}$ de ancho $\times 25 \mathrm{~mm}$ de longitud de acuerdo a la norma ASTM E-08. En este caso, se aplicó una carga uniaxial de 600 kN en una máquina Tinius Olsen Super L290. La evaluación de la prueba de impacto Charpy se realizó a una temperatura de $20^{\circ} \mathrm{C}$ conforme al requerimiento de la especificación ASTM A-370 y ASTM E-23 empleando una máquina Tinius Olsen de $256 \mathrm{~J}$ máx., con un péndulo de $3.08 \mathrm{~kg} / \mathrm{s} \mathrm{de} \mathrm{caída}$ libre. Así mismo, las pruebas de dureza Vickers se llevaron acabo de acuerdo a la norma ASTM E384 empleando un durómetro Future Tech FM7 con una carga de $0.5 \mathrm{~kg}_{\mathrm{f}}$ por $15 \mathrm{~s}$. 
Efecto de la Profundidad sobre la Soldabilidad de Aceros Ferríticos en Ambientes Simulados Unidos por
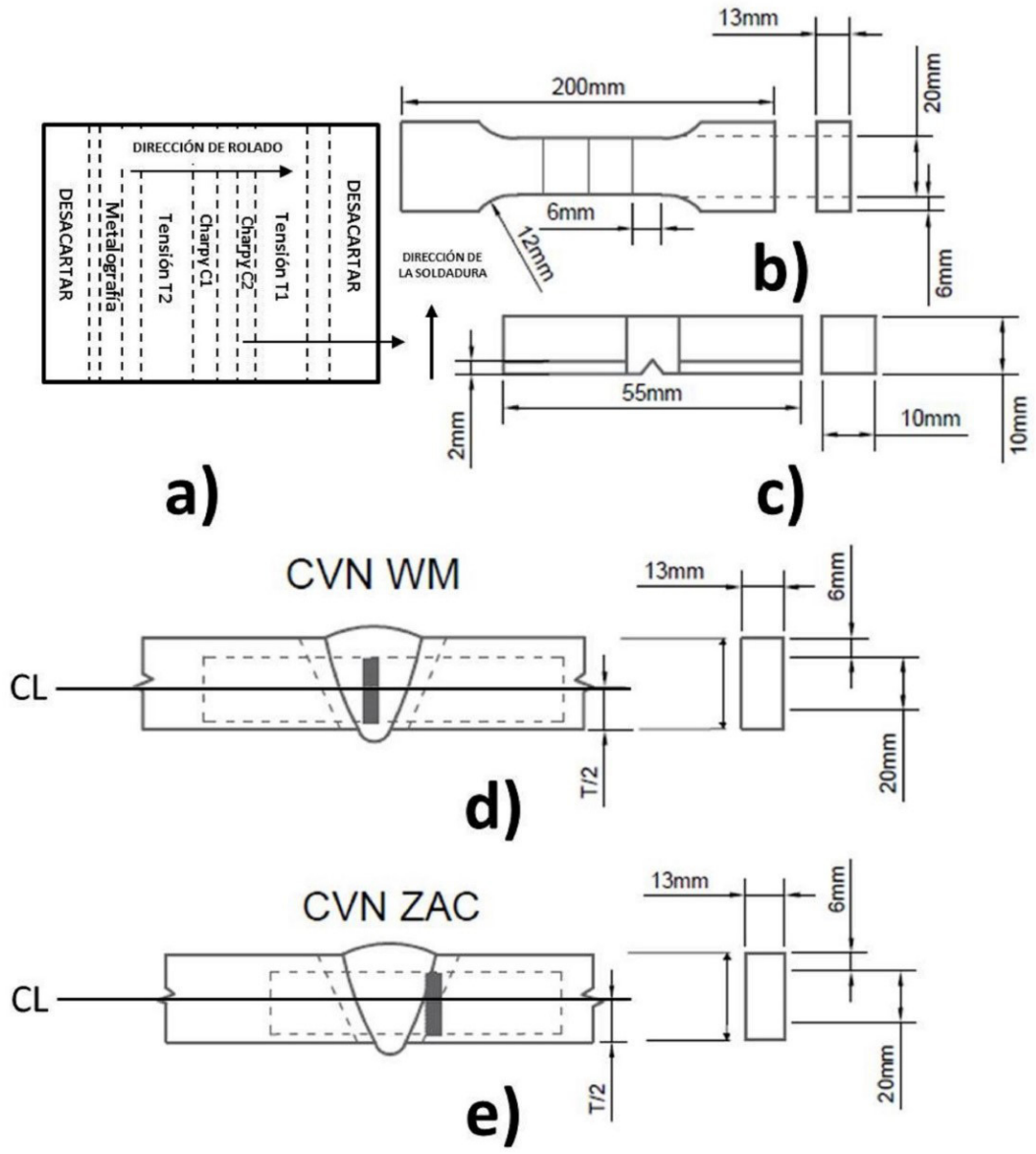

Figura 2. Esquema de las dimensiones de muestras para ensayos mecánicos; (a) cupón de soldadura; (b) espécimen de tensión; (c) espécimen de impacto; (d) localización de muesca en ZF; (e) localización de muesca en zona afectada por el calor ZAC.

Cabe mencionar que todas las muestras ensayadas mecánicamente se inspeccionaron micro estructuralmente de acuerdo a lo descrito en la sección 2.4. Finalmente, se analizó químicamente el cordón de la soldadura en un equipo de Espectrometría de Chispa Marca Belec Modelo Vario Lab de cuerdo a la norma ASTM 415.

\subsection{Caracterización micro estructural y macro ataques de los cordones de soldadura}

Se cortaron cinco muestras de los aceros ASTM A36 soldados a diferentes profundidades y se prepararon metalográficamente mediante desbaste con lijas de 120 a 600 granos por pulgada lineal sobre platos giratorios y enjuagues consecutivos de la superficie para eliminar residuos del material abrasivo, así como limpieza ultrasónica por 10 min., en vasos de precipitado con etanol para remover material incrustado.

En la etapa de pulido se emplearon paños micro cloth, impregnados con alúmina de $0.5 \mu \mathrm{m}$ y finalmente se pulieron a acabado espejo con pasta de diamante de $0.3 \mu \mathrm{m}$ impregnado en un paño micro cloth sobre un plato estático y seguido de una limpieza ultrasónica por $10 \mathrm{~min}$. Posteriormente, las muestras se macro atacaron con una mezcla de Nital al $5 \%$ por $5 \mathrm{~s}$ y se analizaron mediante en un Estereoscopio marca Nikon SMZ 745T modelo C-FMBN 2007934. Otras muestras se revelaron micro estructuralmente con Nital al $5 \%$ por 5 s y se inspeccionaron por medio de un Microscopio Óptico Metalográfico Olympus modelo PMG3 empleando magnificaciones de 50X a 500X con el fin de analizar la microestructura e identificar las fases formadas en las diversas zonas del cordón de soldadura. Así mismo, la descripción micro estructural a altas amplificaciones se observó mediante un Microscopio Electrónico de Barrido (MEB) modelo JEOL JSM-6490LV. 


\section{Resultados y Discusión}

\subsection{Análisis del efecto de la porosidad y composición química en muestras macro atacadas y ensayadas mecánicamente por impacto y tensión uniaxial}

La Figura 3a-d, muestran cortes transversales de los cordones de soldadura macro atacados en función de la profundidad de las soldaduras realizadas a 10, 20, 30 y 40m. Se aprecia que la cantidad de porosidad incrementa a diferentes profundidades debido a la generación de gas en función del aumento de la presión. Por otro lado, se observa que el área de la porosidad se acentúa de menor a mayor desde la raíz hacia el área del refuerzo del cordón de soldadura.
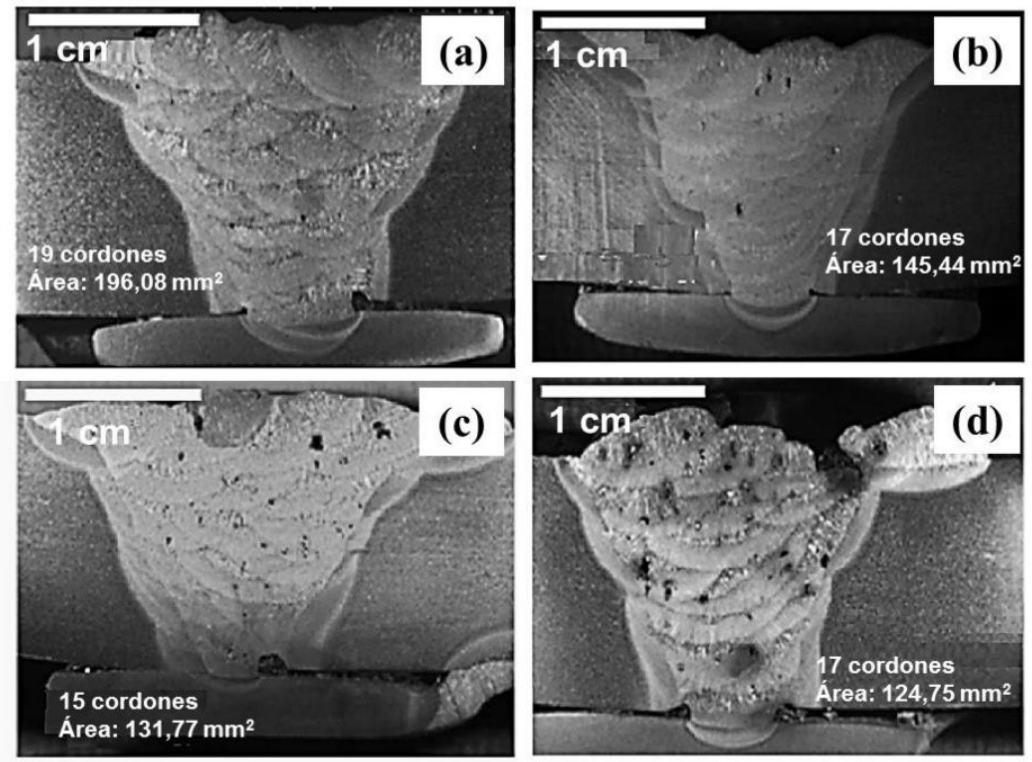

Figura 3. Macrografías de los cordones de soldadura realizados a diferentes profundidades: (a) 10m; (b) 20m; (c) 30m; y (d) 40m. Magnificación 6X.

El incremento del porcentaje de la porosidad en los cordones de soldadura está determinado en función del aumento de la presión sobre el charco de soldadura a diferentes profundidades (ver Tabla 6). Mientras tanto, se aprecia que para un incremento en el porcentaje de porosidad $(0.35 \%)$ se tiene un valor máximo de impacto de $40.7 \mathrm{~J}$ y otro máximo de $82 \mathrm{~J}$ en la ZAC. En contraste, respecto al incremento del porcentaje de porosidad, ambos valores de energía disminuyen a 23.7 y $47 \mathrm{~J}$, respectivamente a $10 \mathrm{~m}$ de profundidad. No obstante, se observa que los valores obtenidos en la ZF y en la ZAC muestran una relación de 2:1 que se encuentran por encima de los criterios descritos por el estándar AWS D3.6M, clase B de $20 \mathrm{~J}$ en promedio y $14 \mathrm{~J}$ el valor mínimo por cada espécimen, determinando que los valores de energía absorbida están más relacionados al tipo de microestructura dúctil obtenidos de valores de calor de entrada de $0.68 \geq 0.94 \mathrm{KJ} / \mathrm{mm}$.

Tabla 6. Porosidad y pruebas de impacto de las probetas soldadas a diferentes profundidades.

\begin{tabular}{|c|c|c|c|c|}
\hline \multirow{2}{*}{$\begin{array}{l}\text { Profundidad } \\
\text { (m) }\end{array}$} & \multirow{2}{*}{$\begin{array}{c}\text { Entrada de calor } \\
\mathrm{HI}^{* * *} \\
(\mathbf{k J} / \mathrm{mm})\end{array}$} & \multirow{2}{*}{$\begin{array}{c}\text { Porosidad } \\
\text { (\%) }\end{array}$} & \multicolumn{2}{|c|}{ Pruebas de impacto } \\
\hline & & & $\begin{array}{l}Z^{*} \\
(J)\end{array}$ & $\begin{array}{c}Z A C^{* *} \\
(J)\end{array}$ \\
\hline 10 & 0,68 & 0,20 & 23,70 & 47,00 \\
\hline 20 & 0,92 & 0,35 & 40,70 & 82,00 \\
\hline 30 & 0,64 & 1,72 & 36,00 & 75,30 \\
\hline 40 & 0,94 & 1,82 & 32,70 & 62,70 \\
\hline
\end{tabular}

*ZF: Zona de Fusión. **ZAC: Zona Afectada por el Calor. ***HI: Calor de entrada (HI: Heat input). 
Efecto de la Profundidad sobre la Soldabilidad de Aceros Ferríticos en Ambientes Simulados Unidos por Soldadura Húmeda

Las propiedades mecánicas de la soldadura húmeda dependen de la profundidad, de los elementos aleantes y de la perdida de estos debido a la oxidación e incremento de la porosidad. En la Figura 4 se aprecia que la muestra con $0.35 \%$ de porosidad tiene un valor máximo promedio de resistencia última a la tensión (UTS) de 494.5 MPa y es consistente con el valor máximo de impacto de $40.7 \mathrm{~J}$. El incremento de la porosidad disminuye las propiedades mecánicas de tensión hasta alcanzar un valor promedio de $425 \mathrm{MPa}$ con un valor de impacto de $32.7 \mathrm{~J}$ con $1.82 \%$ de porosidad. Esta disminución en propiedades mecánicas por efecto de la porosidad es confirmado por Pessoa et al. [14]. Esto es debido a la coalescencia y al crecimiento de la porosidad durante el flujo plástico [15]. Además, se asocia a la disminución del UTS, al decremento en contenido de los elementos de Mn y C en función de la profundidad, ya que estos elementos endurecen el acero (C) e incrementan la resistencia a la tenacidad (Mn) siendo gammágenos (Tabla 7) [16]. Sin embargo, en la literatura [17] se sugiere que la adición de Ni y Mo mejora la resistencia mecánica y la tenacidad en soldaduras húmedas.

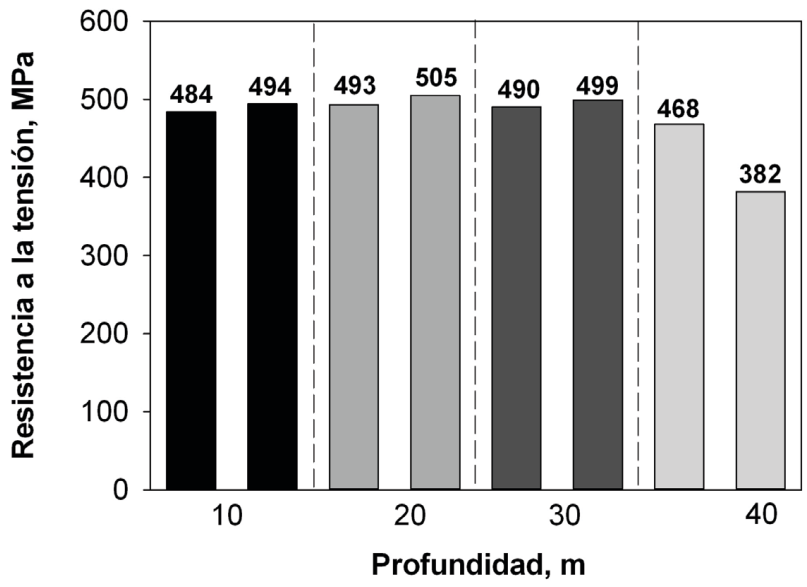

Figura 4. Resultados de las pruebas de tensión en relación con la profundidad en cupones soldados con el proceso MMAW.

Tabla 7. Análisis químico en la zona de fusión.

\begin{tabular}{|c|c|c|c|c|c|c|c|c|c|c|c|c|c|c|}
\hline No. & $\begin{array}{l}P^{*} \\
\text { (m) }\end{array}$ & C & $S$ & Mn & $\mathbf{P}$ & Si & $\mathrm{Cr}$ & $\mathbf{N i}$ & Mo & $\mathrm{Cu}$ & v & $\mathbf{N b}$ & Ti & $\mathbf{w}$ \\
\hline 1 & 10 & 0,190 & 0,009 & 0,710 & 0,007 & 0,170 & 0,060 & 0,040 & 0,010 & 0,056 & 0,040 & 0,004 & 0,004 & 0,002 \\
\hline 2 & 20 & 0,180 & 0,010 & 0,670 & 0,007 & 0,200 & 0,050 & 0,040 & 0,010 & 0,058 & 0,040 & 0,004 & 0,004 & 0,002 \\
\hline 3 & 30 & 0,090 & 0,010 & 0,360 & 0,011 & 0,320 & 0,020 & 0,040 & 0,020 & 0,067 & 0,040 & 0,004 & 0,018 & 0,002 \\
\hline 4 & 40 & 0,090 & 0,009 & 0,290 & 0,011 & 0,390 & 0,020 & 0,040 & 0,020 & 0,066 & 0,040 & 0,004 & 0,018 & 0,002 \\
\hline
\end{tabular}

* Profundidad.

Se postula que la pérdida del contenido de Mn por oxidación está directamente relacionada con la presión parcial del $\mathrm{O}$ en función de la profundidad y en el caso del $\mathrm{C}$ con la formación de $\mathrm{CO}_{2(\mathrm{~g})}$ [18]. Por otro lado, incrementa considerablemente la cantidad de $\mathrm{H}$ y la afinidad con el $\mathrm{O}$ promoviendo la formación de porosidades reduciendo el área efectiva de la sección transversal de la unión soldada y por consecuencia la resistencia a la tensión.

Por otro lado, Ibarra et al. [19], sugieren que el contenido de Mn disminuye en el metal de soldadura con la profundidad e incrementa el Si. Cabe mencionar que el elemento de Si promueve ligeramente la fase austenítica y en veces la fase de ferrita, el cual es desoxidante y endurecedor.

\subsection{Evaluación del cordón de soldadura por Micro dureza Vickers}

En la Figura 5 se muestra la variación de la micro dureza Vickers en el cordón de soldadura en función de la aplicación de la soldadura húmeda a diferentes profundidades. 


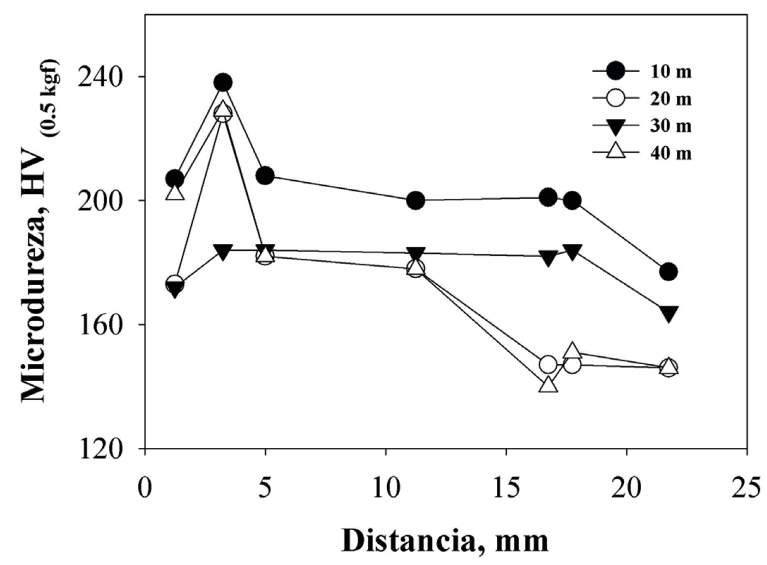

Figura 5. Variación de la micro dureza Vickers en el cordón de soldadura en función de la aplicación de la soldadura a diferentes profundidades.

En la Figura 6 se observan diferentes micro durezas HV medidas a diferentes distancias en las zonas del cordón de soldadura. Se aprecia que el valor máximo de micro dureza $\left(278 \mathrm{HV}_{0.5 \mathrm{kgf}}\right)$ se obtiene en la ZAC/LF y un valor mínimo en la ZF (120 $\left.\mathrm{HV}_{0.5 \mathrm{kgf}}\right)$. Sin embargo, las bajas micro durezas se obtienen a profundidades de $40 \mathrm{~m}$ debido al incremento de la fase ferrítica de baja temperatura y zonas de estas fases recristalizadas. Este descenso de las micro durezas se reportan a la formación de fases de baja dureza Terán et al. [20], ya que el incremento de la potencia en la soldadura favorece la recristalización de fases de baja dureza. Esto está asociado con una alta entrada de calor debido a una baja velocidad de avance, la cual genera la formación de nuevos granos que son enfriados rápidamente.

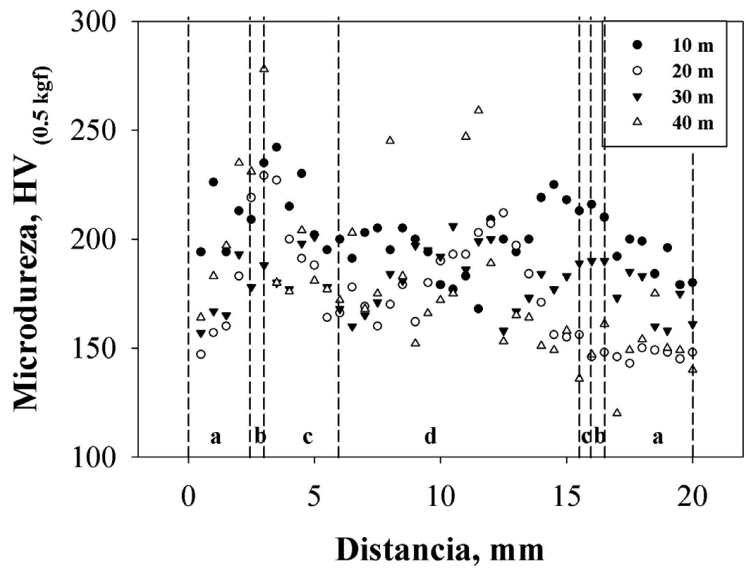

Figura 6. Dureza Vickers en función de las distancias en las zonas del cordón de soldadura y aplicación de la soldadura a diferentes profundidades. (a) MB: Metal base; (b) LF: Línea de fusión; (c) ZAC: Zona afectada por el calor; (d) MA: Metal de aporte.

\subsection{Evolución micro estructural en el cordón de la soldadura}

Básicamente, las fases identificadas y cuantificadas provienen de la transformación de fase de la $y$ a una variedad de ferritas (F), ferrita al límite de grano (FLG), ferrita Widmanstatten Secundaria (FW), ferrita alotriomórfica (AF) y ferrita acicular (FA), martensita atérmica (MA) y transformaciones masivas de vainita superior e inferior (BS y BI). En la Tabla 8 se resume el porcentaje de fases en función de la profundidad de la soldadura húmeda. Se observa que la ferrita (F) de baja temperatura incrementa de 75.32 a $95.04 \%$ a profundidades de 10 a $30 \mathrm{~m}$. Mientras tanto la FW disminuye hasta los $40 \mathrm{~m}$. Se postula que esta fase se origina a altas temperaturas y a velocidades de enfriamiento intermedias por debajo de la línea de transformación $\mathrm{A}_{3}$. En consecuencia, mitiga el crecimiento de la AF. 
Efecto de la Profundidad sobre la Soldabilidad de Aceros Ferríticos en Ambientes Simulados Unidos por Soldadura Húmeda

Tabla 8. Porcentaje de fases presentes a diferentes profundidades de soldadura húmeda.

\begin{tabular}{|c|c|c|c|c|c|c|}
\hline \multirow{2}{*}{$\begin{array}{l}\text { Profundidad } \\
\text { (m) }\end{array}$} & \multicolumn{6}{|c|}{$\begin{array}{l}\text { Fases presentes en diferentes profundidades } \\
\text { (\%) }\end{array}$} \\
\hline & $\mathbf{F}$ & FW & FA & FLG & $\mathbf{M}$ & B \\
\hline 10 & 75,32 & 17,33 & 4,78 & 2,57 & 0 & 0 \\
\hline 20 & 73,39 & 3,39 & 17,13 & 5,86 & 0 & 0,21 \\
\hline 30 & 95,04 & 1,63 & 2,97 & 0 & 0 & 0,37 \\
\hline 40 & 83,57 & 0,35 & 16,09 & 0 & 0 & 0 \\
\hline
\end{tabular}

(F) Ferrita; (FW) Ferrita Widmanstatten Secundaria; (FA) Ferrita Acicular; (FLG) Ferrita al límite de grano; (M) Martensita; y (B) Bainita.

Este resultado es consistente con la entrada de calor (ver Tabla 6) y la disminución del contenido de los elementos de $\mathrm{Mn}$ y C que son elementos que amplían la región de formación de la y (ver Tabla 7). En contraste, el porcentaje de fase de la FA es variante, ya que depende del nivel de inclusiones y el tamaño de grano austenítico. Finalmente, la bainita superior e inferior es una fase difícil de identificar y solamente se cuantificó por coloración a profundidades de soldaduras aplicadas de 20 y $30 \mathrm{~m}$. El mismo problema se tiene para la MA. Estas fases se identificaron en la ZF y la ZAC (ver Figura 7 y 8 ).
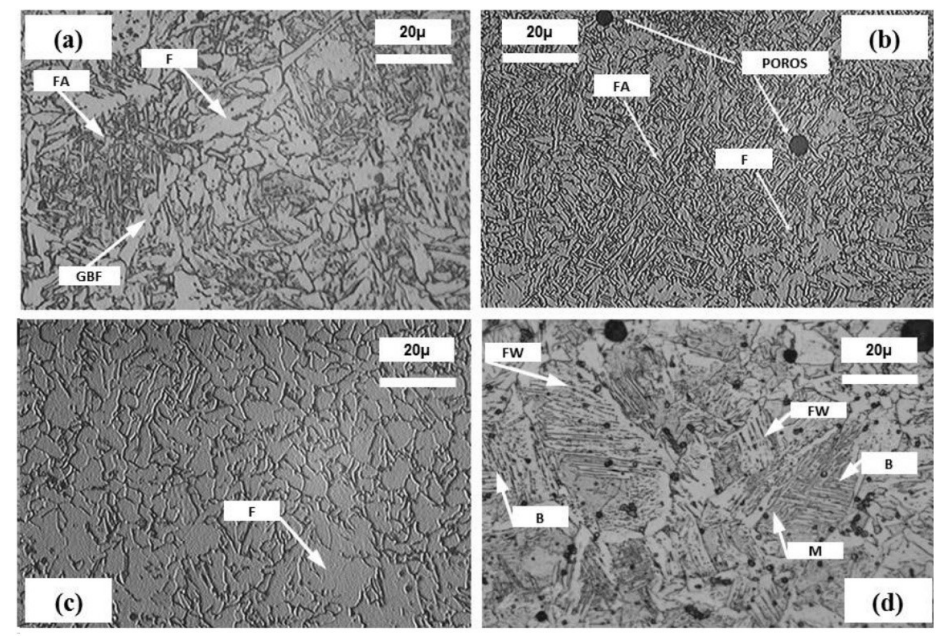

Figura 7. Micrografías de la zona de fusión de las probetas soldadas a profundidades: (a) $10 \mathrm{~m}$; (b) $20 \mathrm{~m}$; (c) $30 \mathrm{~m}$; y (d) $40 \mathrm{~m}$.
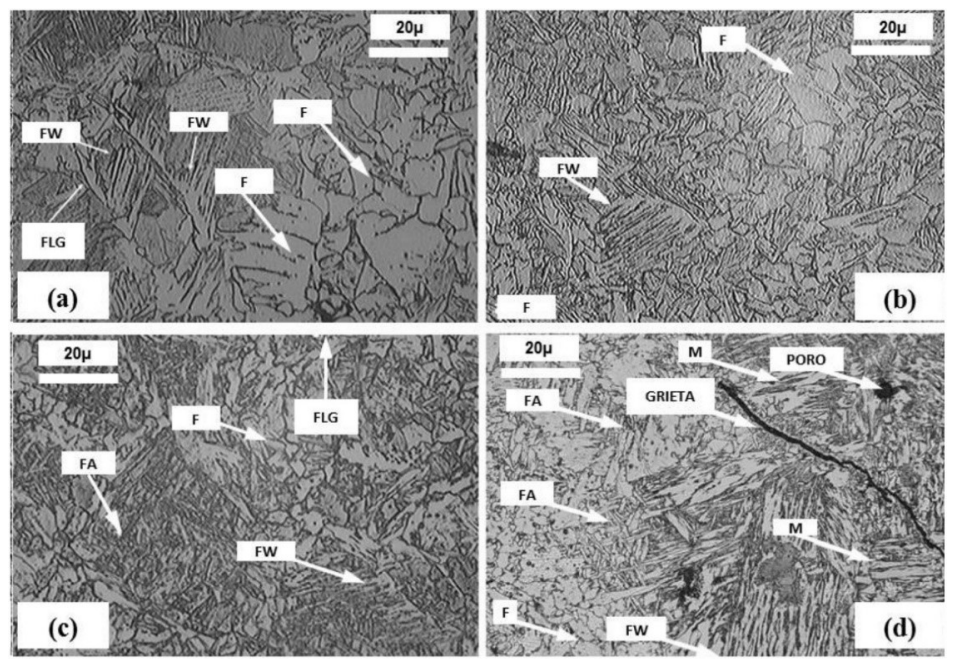

Figura 8. Micrografías de la zona afectada por el calor de las probetas soldadas a profundidades: (a) $10 \mathrm{~m}$; (b) $20 \mathrm{~m}$; (c) $30 \mathrm{~m}$; y (d) $40 \mathrm{~m}$. 
En la literatura [21,22] sugieren que las ferritas aciculares en la ZAC y ZF mejoran las propiedades mecánicas. Debido a que el tipo de morfología frena o desvía la trayectoria de las micro grietas. Estas fases se correlacionan con el valor de $40.7 \mathrm{~J}$ de tenacidad en la ZF y $82 \mathrm{~J}$ en la ZAC a 20m como se muestran en la Tabla 6.

Estas fases se aprecian en mayor cantidad en la ZF a $20 \mathrm{~m}$ y en la ZAC a $30 \mathrm{~m}$ (Figuras $7 \mathrm{~b}$ y $8 \mathrm{c}$. Por otro lado, en la ZF y ZAC se observan regiones ricas en martensita a $40 \mathrm{~m}$ (Figuras $7 \mathrm{~d}$ y $8 \mathrm{~d}$ ). Cabe mencionar que las fases martensíticas atérmicas disminuyen las propiedades mecánicas de resistencia y tenacidad, y durante la solidificación se generan micro grietas debido a la formación de esfuerzos residuales que la misma fase induce en el material. Este efecto combinado a 40m disminuyen las propiedades mecánicas (Figura 4). En la ZAC algunas regiones de granos ferríticos recristalizados en las soldaduras aplicadas a profundidades de $40 \mathrm{~m}$ se muestran en la Figura $8 \mathrm{~d}$.

Generalmente, las soldaduras aplicadas bajo el agua o a la intemperie dependen de la formación de una serie de fases fuera de equilibrio en la ZAC y que tienen un rol significativo en las propiedades mecánicas. A altas amplificaciones se aprecian las morfologías y distribuciones de las fases en las ZF y ZAC (Figuras 9 y 10). Se observa la martensita atérmica en forma de placas y bainita inferior con placas finas, así como algunas regiones de la FWS. Las micro porosidades son más notorias en la ZF.
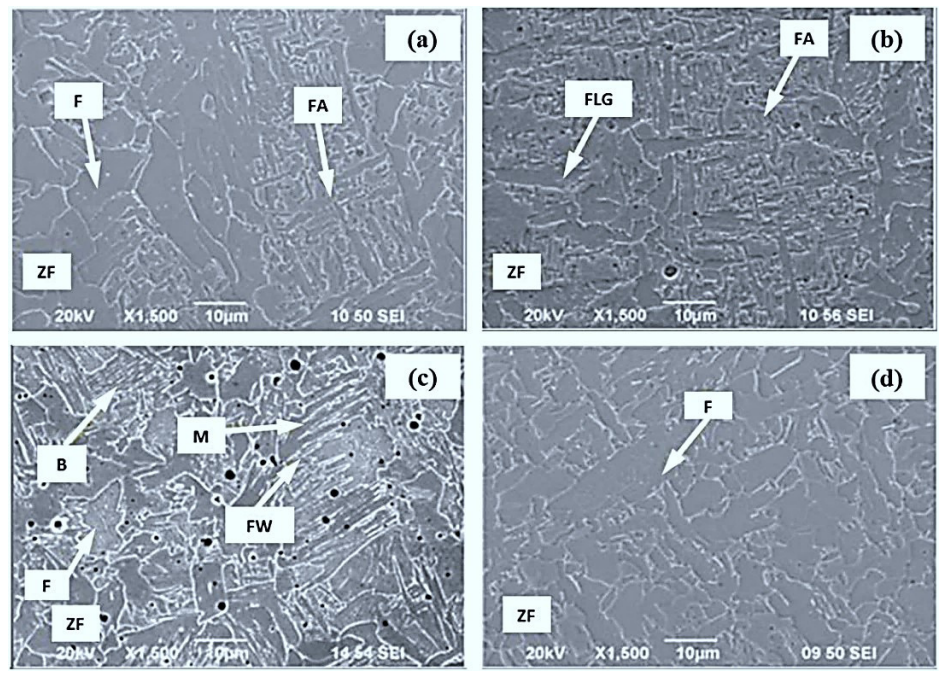

Figura 9. Imágenes de electrones secundarios de la zona de fusión (ZF) de soldadura aplicadas a diferentes profundidades: (a) 10m; (b) 20m; (c) $30 \mathrm{~m}$; y (d) $40 \mathrm{~m}$.

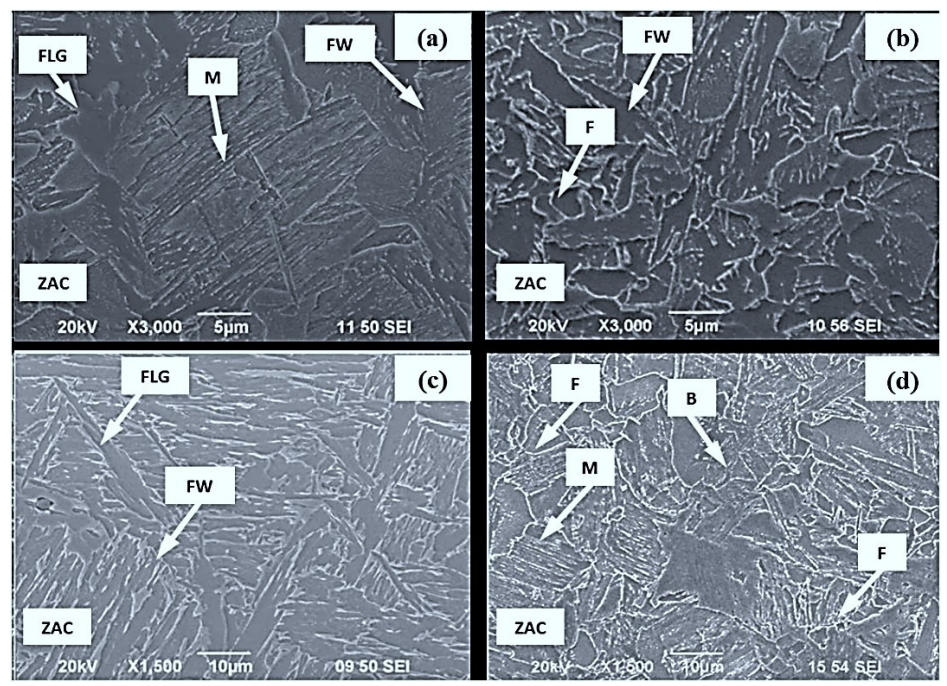

Figura 10. Imágenes de electrones secundarios de la zona afectada por el calor (ZAC) de soldadura aplicadas a diferentes profundidades: (a) 10m; (b) 20m; (c) $30 \mathrm{~m}$; y (d) $40 \mathrm{~m}$. 
Efecto de la Profundidad sobre la Soldabilidad de Aceros Ferríticos en Ambientes Simulados Unidos por Soldadura Húmeda

\section{Conclusiones}

En base a los resultados obtenidos en el presente estudio realizado del efecto de la profundidad sobre la soldabilidad de aceros ferríticos en ambientes simulados unidos por soldadura húmeda, las conclusiones son las siguientes:

1)La variación del análisis cuantitativo de las micro durezas está relacionada con la heterogeneidad de la microestructura. Mientras que el porcentaje de la porosidad incrementa en función de la profundidad de la aplicación de la soldadura. Debido a la generación de gases en el charco líquido que quedan atrapados una vez que solidifica el cordón soldado.

2)Se postula que las mejores propiedades mecánicas y metalúrgicas se obtuvieron a $20 \mathrm{~m}$ de profundidad al obtener la cantidad adecuada de subenfriamiento compensando con un mayor porcentaje de FA.

3)Se postula que la disminución de las propiedades mecánicas en la unión soldada se obtuvieron a 40m de profundidad debido a la pérdida del contenido de Mn, asimismo por la formación de porosidades que reduce el área efectiva de la sección transversal y por consecuencia afectando la resistencia a la tensión.

4)Todas las uniones realizadas, presentan excelentes propiedades mecánicas (resistencia, tenacidad y dureza) cumpliendo con los criterios de aceptación del estándar AWS D3.6M para soldadura clase B.

\section{Agradecimientos}

Los autores agradecen a la compañía constructora Subacuática Diavaz por las facilidades otorgadas para la realización de los cupones soldados bajo el agua y al grupo de colaboradores de investigación y desarrollo en buceo industrial.

\section{Referencias}

[1] Scott, AD, Bracarense AQ, González LP, Pessoa ECP, Puchol $R Q$, Pérez MR. Caracterización metalográfica de depósitos de soldadura subacuatica mojada. Cuba: [publisher unknown]; $2008.6 \mathrm{p}$.

[2] Ilker Yelbay H, Ibrahim Cam C, Hakan G. Non-destructive determination of residual strees state in steel weldments by Magnetic Barkhausen Noise technique. NDT \& E International. 2010;43(1):29-33

[3] Liu S. Maintenance and repair welding in the open sea. Welding Journal. 2005;84(11):54-59.

[4] Christensen N. The metallurgy of underwater welding. New York: [publisher unknown]; 2005.

[5] Nakpradit T, Poopat B. Investigation of diffusible hydrogen content and microstructure examination of underwater welding. International Journal of Applied Science and Technology. 2010;3(3):45-51.

[6] Ibarra S, Grubbs C, Olson D. The nature of metallurgical reaction in underwater wet welding. In: Proceedings of the 19th Nanual ofshore. Houston: Offshore Technology Corp.; 1987. 26 p. Technology Conference Report.

[7] Silva RJ. Efeito dos parâmetros de soldagem na formação do cordão [tesis de maestría]. Botucatu: Faculdade de Mecânica, Universidade Estadual Paulista; 1995.

[8] Rowe M, Liu S. Final report MT-CWJCR-099-032. [place unknown]: Global Industries; 1999.

[9] Rowe MD, Reynolds TJ. The effect of ferro-alloy additions and depth on the quality of underwater wet welds. Welding Journal. 2002;81(8):156-166.

[10] Sindo K. Welding metallurgy. United States of America: Wiley; 2003. 34 p.

[11] American Society for Testing and Materials. ASTM A36/A36M-14: specification for carbon structural steel. West Conshohocken: ASTM; 2014.

[12] American Welding Society. AWS A5.1/A5.1M: specification for carbon steel electrodes for shielded metal arc welding. Washington: AWS; 2012

[13] American Welding Society. Underwater Welding Code D3.6M. 5th ed. Washington: AWS; 2010. 37 p.

[14] Pessoa ECP, Bracarense AQ, Liu S, Guerrero FP, Zica EM Porosity variation along multipass underwater wet welds and its influence on mechanical properties. Journal of Materials Processing Technology. 2006;179(1-3):239-243. http://dx.doi. org/10.1016/j.jmatprotec.2006.03.071.

[15] George E. Dieter, mechanical metallurgy. 3rd ed. New York: McGraw-Hill; 1981.

[16] Ando S, Asahani TA. Study on the metallurgical properties of steel welds with underwater gravity welding. In: Underwater Welding: Proceedings of the International Conference held at Trondheim. Norway: International Institute of Welding; 1983.

[17] Santos MJ, Monteiro FC, Rizzo AQ. Bracarense, development of an oxyrutile electrode for wet welding. Supplement to the Welding Journal. 2012;91:319-328.

[18] Lindhorst L. On the effects of wet underwater welding on the fracture behaviour of Welds. In: Proceedings of the 16th International Conference on Structural Mechanics In Reactor Technology. Washington: [publisher unknown]; 2001.

[19] Ibarra S, Grubbs CES, Liu S. State of the art and practice of underwater wet welding of steel. In: Proceedings: International Workshop on Underwater Welding of Marine Structures. Louisiana: [publisher unknown]; 1994. p. 49-67. 
[20] Terán G, Cuamatzi-Meléndez R, Albiter A, Maldonado C, Bracarense AQ. Characterization of the mechanical properties and structural integrity of T-welded connections repaired by grinding and wet welding. Materials Science and Engineering. 2014;599:105-115. http://dx.doi.org/10.1016/j.msea.2014.01.078.
[21] Rowe M, Liu S. Recent developments in underwater wet welding. Science and Technology of Welding and Joining. 2001;6(6):387396. http://dx.doi.org/10.1179/stw.2001.6.6.387.

[22] Bhadeshia HKDH. Bainite in steel, transformations, microstructure and properties. London: IOM; 2001. p. 237-276. 